\title{
A realist review of mobile phone-based health interventions for non-communicable disease management in sub-Saharan Africa
}

\author{
Daniel Opoku*, Victor Stephani and Wilm Quentin
}

\begin{abstract}
Background: The prevalence of non-communicable diseases (NCDs) is increasing in sub-Saharan Africa. At the same time, the use of mobile phones is rising, expanding the opportunities for the implementation of mobile phone-based health (mHealth) interventions. This review aims to understand how, why, for whom, and in what circumstances mHealth interventions against NCDs improve treatment and care in sub-Saharan Africa.
\end{abstract}

Methods: Four main databases (PubMed, Cochrane Library, Web of Science, and Google Scholar) and references of included articles were searched for studies reporting effects of mHealth interventions on patients with NCDs in sub-Saharan Africa. All studies published up until May 2015 were included in the review. Following a realist review approach, middle-range theories were identified and integrated into a Framework for Understanding the Contribution of mHealth Interventions to Improved Access to Care for patients with NCDs in sub-Saharan Africa. The main indicators of the framework consist of predisposing characteristics, needs, enabling resources, perceived usefulness, and perceived ease of use. Studies were analyzed in depth to populate the framework.

Results: The search identified 6137 titles for screening, of which 20 were retained for the realist synthesis. The contribution of mHealth interventions to improved treatment and care is that they facilitate (remote) access to previously unavailable (specialized) services. Three contextual factors (predisposing characteristics, needs, and enabling resources) influence if patients and providers believe that mHealth interventions are useful and easy to use. Only if they believe mHealth to be useful and easy to use, will mHealth ultimately contribute to improved access to care. The analysis of included studies showed that the most important predisposing characteristics are a positive attitude and a common language of communication. The most relevant needs are a high burden of disease and a lack of capacity of first-contact providers. Essential enabling resources are the availability of a stable communications network, accessible maintenance services, and regulatory policies.

Conclusions: Policy makers and program managers should consider predisposing characteristics and needs of patients and providers as well as the necessary enabling resources prior to the introduction of an mHealth intervention. Researchers would benefit from placing greater attention on the context in which mHealth interventions are being implemented instead of focusing (too strongly) on the technical aspects of these interventions.

Keywords: mHealth, Mobile phone, Non-communicable diseases, Chronic diseases, Sub-Saharan Africa, Realist review, Health policy

\footnotetext{
* Correspondence: daniel.opoku@campus.tu-berlin.de

Department of Healthcare Management, Technische Universität Berlin, Straße des 17. Juni 135, Sekretariat H80, 10623 Berlin, Germany
}

(c) The Author(s). 2017 Open Access This article is distributed under the terms of the Creative Commons Attribution 4.0 International License (http://creativecommons.org/licenses/by/4.0/), which permits unrestricted use, distribution, and reproduction in any medium, provided you give appropriate credit to the original author(s) and the source, provide a link to the Creative Commons license, and indicate if changes were made. The Creative Commons Public Domain Dedication waiver (http://creativecommons.org/publicdomain/zero/1.0/) applies to the data made available in this article, unless otherwise stated. 


\section{Background}

In sub-Saharan Africa (SSA), the prevalence of noncommunicable diseases (NCDs) is increasing rapidly [1], placing a growing burden on already weak health systems in the region $[2,3]$. At the same time, the use of mobile phones is continuously rising, expanding the opportunities for the implementation of mobile phonebased health interventions (mHealth interventions) [4-6]. The World Health Organization (WHO) has proposed the further development and more widespread use of mHealth interventions for the prevention, management, and treatment of NCDs and their risk factors as part of its Global Action Plan for the prevention and control of NCDs [7].

In fact, mHealth interventions are increasingly used in low- and middle-income countries, including those in SSA [8]. Three recent systematic reviews, two specifically focusing on the use of mHealth interventions for the care and management of NCDs in SSA [6] and in developing countries [9], and the other looking more broadly at the use of mHealth interventions against chronic diseases in developing countries [10], found that the included studies generally reported positive outcomes. However, the authors also noted that there was insufficient evidence to support the scale-up of mHealth interventions because there were only five studies from SSA countries [6] and only nine studies from developing countries $[9,10]$. In addition, the authors highlighted that further research was needed to better understand the causal pathways linking mHealth to improved care for patients with NCDs [6].

Traditional systematic reviews, which are often focused on randomized controlled trials, usually do not allow one to uncover causal pathways or to identify contextual mechanisms that may explain whether, why, and how interventions might work [11]. Realist reviews have emerged as an alternative method for systematic reviews, aiming to provide answers for policy makers about the causal mechanisms that link context, intervention, and outcomes [12].

Understanding these mechanisms is particularly relevant for complex interventions, such as mHealth interventions, which are implemented in vastly different healthcare settings (varying from rural communities $[13,14]$ to major university hospitals [15]), use various functions of mobile phones (from text messaging [16-18] to picture transmission [19]), target widely different health conditions (from skin lesions [20] to maternal health [21, 22]), and are put to use by persons with very different backgrounds, behaviors, skills, and beliefs [23-25].

This review aimed to understand how, why, for whom, and in what circumstances mHealth interventions contribute to improved treatment and care for patients with NCDs. More precisely, the first question ("how?") that the review aimed to answer was: What is the specific contribution that mHealth makes to patient treatment and care? As the review proceeded, it became clear that the main contribution of mHealth interventions is that they facilitate (remote) access to previously unavailable and often specialized - services. Therefore, the objective of this review was to answer the following specific questions: (1) What are the causal mechanisms ("why?") that explain if an mHealth intervention facilitates access to care? (2) How do patient and provider characteristics ("for whom?") influence these mechanisms? (3) What is the influence of contextual factors ("what circumstances?") on these mechanisms?

\section{Methods}

This review followed guidelines for realist reviews $[11,12,26,27]$ because the research questions could not be answered using more traditional forms of systematic reviews. Realist reviews focus on identifying (middle-range) theories, which can provide guidance to the available literature. These theories then help us to understand the mechanisms that explain why an intervention has worked in one context but not in another. However, such Context-Mechanism-Outcome (C-M-O) relationships identified in realist reviews do not imply that a specific context will always lead to a specified outcome. Instead, realist reviews assume that outcomes are the result of choices made by individuals whose interactions are influenced by the intervention and by the context of implementation [12, 26, 27]. (See Table 1 for the operational definition of the $\mathrm{C}-\mathrm{M}-\mathrm{O}$ model of hypotheses adapted in this review.)

\section{Scoping the literature and searching for relevant studies}

An initial scoping review was conducted to identify candidate theories (see below) and to obtain a broad overview of the available literature on mHealth interventions aiming to improve treatment and care for patients with NCDs in SSA. Following this initial search, the review question was progressively refined to focus more specifically on the contribution of mHealth to facilitating access to previously unavailable care.

A search strategy was developed, using various combinations of the following search terms: "mHealth", "non-communicable diseases", and "sub-Saharan Africa". PubMed, Cochrane Library, Web of Science, and Google Scholars, were searched and re-searched from March to May 2015. (Additional file 1 provides details of the search strategies developed for the four databases.) In addition, a hand search was performed of the Journal of Telemedicine and Telecare, the Journal of Telemedicine and e-Health, and of reference lists of screened studies and existing reviews.

\section{Inclusion and exclusion criteria}

The review included various study designs (randomized controlled trials, mixed methods, and qualitative interview 
Table 1 Operational definition of the C-M-O model of hypotheses adapted in this review

\begin{tabular}{ll}
\hline C-M-O & Operational definition \\
\hline Context & This is defined as the prevailing conditions and circumstances within which patients and/or healthcare providers behave \\
& or decide to use mobile phone-based health interventions for the treatment and care of non-communicable diseases in \\
& sub-Saharan Africa. For example: \\
& - Patient/provider predisposing characteristics (age, gender, etc.) \\
& - Patient/provider needs \\
& - Patient/provider enabling resources \\
& The factors or active "ingredients" of a mobile phone-based health intervention which directly/indirectly influence both \\
& intended and unintended health outcomes and/or outputs of the treatment and care of non-communicable diseases in \\
& a well-defined context in sub-Saharan Africa. For example: \\
& - How easy to use the patients and healthcare providers find the mobile technology involved in the intervention \\
& - How useful patients and healthcare providers perceive the mHealth intervention to be over alternative programs and \\
& forms of accessing healthcare \\
& This constitutes the sustained use of mHealth interventions and - in turn — better patient access to care \\
Outcome &
\end{tabular}

studies) and publication types (peer-reviewed articles, gray literature, and other forms of research reports). Titles, keywords, and abstracts were screened by the corresponding author (DO) to identify relevant studies based on a set of inclusion criteria developed during the initial scoping review. A second reviewer (VS) also independently screened retrieved studies. If there was disagreement between reviewers, studies were retained for full-text screening. The following inclusion criteria were applied: (1) studies took place in sub-Saharan Africa (i.e., in at least one of the 47 countries in the WHO African region), (2) interventions relied on the use of (mobile) phones, (3) studies focused on NCD-related treatment and care, and (4) studies provided an evaluation of the relationship between the intervention and NCD care. No language restrictions or time limits were applied.

Full-texts of 126 studies were retrieved and independently screened by DO and VS. At this stage, studies were excluded if interventions were based on phones and not primarily on mobile phones. In case of doubts, corresponding authors of studies were consulted for clarification. Studies were also excluded if they did not report results of (clinical) outcomes and/or an assessment of the intervention by patients, professionals, or proxies (e.g., relatives or guardians). In case of disagreements between DO and VS on the eligibility of studies, these were resolved by WQ.

\section{Identifying candidate theories}

During the initial scoping review, a number of candidate theories with potential explanatory value for mHealth interventions were explored. The identified theories and models included the Middle-Range Theory of Self-Care of Chronic Illness [28], the Theory of Reasoned Action/ Theory of Planned Behavior [29], Rosenstock's Health Belief Model [30], Andersen's Behavioral Model of Health Services Utilization [31, 32], Young's ChoiceMaking Model [33], and Davis's Technology Acceptance Model [34, 35]. (See Additional file 2 for the reasons of inclusion/exclusion.)
Following discussions within the review team, Andersen's Behavioral Model of Health Services Utilization was retained because it could potentially provide insights into the mechanisms linking contextual and individual level factors with improved access to care. According to Andersen's model, peoples' decisions to use (or access) healthcare services are determined by three main factors: (1) predisposing characteristics (e.g., age, health beliefs), (2) enabling resources (e.g., availability of providers), and (3) need (e.g., burden of disease) [32].

As the review proceeded, Davis's Technology Acceptance Model was found to provide additional insights into mechanisms that are important for explaining improved access to care through mHealth interventions. Davis's Technology Acceptance Model posits that the use and acceptance of technology is determined by two factors: perceived usefulness and perceived ease of use. According to Davis's theory, health professionals will perceive a technology to be useful if they believe that it will help them to do a better job, and they will perceive a technology to be easy to use if they believe that it can be used without effort [35].

\section{Data extraction, analysis, and synthesis}

Two data extraction templates were developed using Excel to collate information on the included studies for analysis and synthesis. One template was used to summarize the characteristics of included studies (author(s), year of publication, title, study design, and country where the study took place). The other template for results and synthesis mainly contained information on the (type of) intervention, modality of interaction, outcome/outputs, and the five categories of the theoretical model: predisposing characteristics, enabling resources, need, perceived usefulness, and perceived ease of use.

The data synthesis involved team discussions in relation to whether the information extracted was rightly placed in the various domains and adjusted accordingly. Common themes were highlighted, examined, and refined in the light of their theoretical contributions. This involved classifying 
findings from different studies into the categories of the theoretical model in order to understand the ContextMechanism-Outcome (C-M-O) relationship. For example, if a study reported that older age groups were more likely to make use of an intervention because they found it more useful than younger age groups, this finding was classified into the category of a predisposing characteristic that leads to perceived usefulness.

\section{Results}

\section{Search results and study characteristics}

A total number of 6201 citations were retrieved, out of which 6181 were excluded after the appraisal process displayed in Fig. 1. The raw inter-rater agreement between DO and VS was 97\% (123/126) after full-text screening. Additional file 3 provides information on key characteristics of the 20 included studies. The studies were published between 2005 and 2015, and presented information on 18 interventions in various areas of care (dermatology, mental healthcare, cancer, diabetes, and hypertension).

\section{The contribution of mHealth to improved treatment and care for patients with NCDs}

The main contribution of mHealth interventions to improved treatment and care for patients with NCDs in SSA countries is that they facilitate (remote) access to previously unavailable - and often specialized - services. In fact, almost all included studies highlighted this characteristic feature of mHealth interventions [20, 36-51].

However, the configuration of mHealth interventions differed considerably across settings, concerning involved actors and the mechanisms through which they facilitated access to care. In 12 studies, mHealth interventions essentially consisted of mobile phone-based consultations between two healthcare providers, where a specialized provider could be reached by another

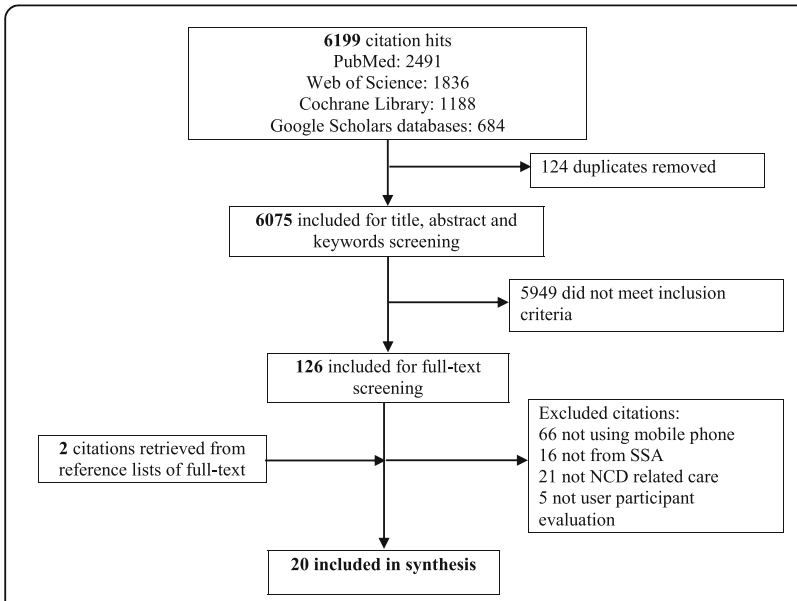

Fig. 1 Flowchart displaying the search results and the screening process for the primary studies provider, thus indirectly improving patient access to specialized care [36, 38-43, 45, 47-49, 52]. In 8 studies, mHealth interventions connected a patient to a provider, thus directly facilitating patient access to (professional) care [20, 37, 46, 50, 51, 53-55].

Participating patients or providers usually required only a few days of training on how to use the mobile technology (such as the mobile phone and its application software) and the consultation procedures [41, 43, 46-48, 50, 55]. An important feature of most mHealth interventions was that interactions between participants usually took place on the basis of standardized information exchange protocols [36, 39, 40, 42, 43, 45-47, 49, 52, 54]. These protocols helped to establish the purpose of the consultations and contributed to systematically ascertaining symptoms, diagnoses, and treatment. (See Additional file 3 for further details.)

\section{From candidate theories toward a framework for understanding mHealth interventions}

During the early stages of the review, Andersen's model and his conceptualization of predisposing characteristics, enabling resources, and need helped to focus the analysis on the role of the context in explaining why mHealth interventions contribute to improved access for some patients and in some areas but not in others. However, as the review proceeded, it became increasingly clear that the context has only an indirect influence on access to health services facilitated by mHealth interventions. At this stage, Davis's Technology Acceptance Model and his conceptualization of perceived usefulness and perceived ease of use contributed to understanding the mechanisms that link the context to improved mHealth based access to healthcare.

The two models of Andersen and Davis were then integrated into a framework for understanding the contribution of mHealth interventions to improved access to care for patients with NCDs in SSA. The framework is illustrated in Fig. 2 and shows that mHealth consultations take place either between a patient and a provider or between two providers with one provider facilitating patient access to another provider with certain specialized skills. The most important patient context factors (predisposing characteristics, enabling factors, and need) are shown on the left-hand side of the figure, while the most important context factors for (specialized) providers are shown on the right-hand side. For providers facilitating access between patients and (specialized) providers, context factors are sometimes more similar to those of patients and sometimes more similar to those of (specialized) providers.

In the center of the figure, arrows indicate the C-M-O relationship: The context factors influence the perceptions of patients and providers concerning how useful 


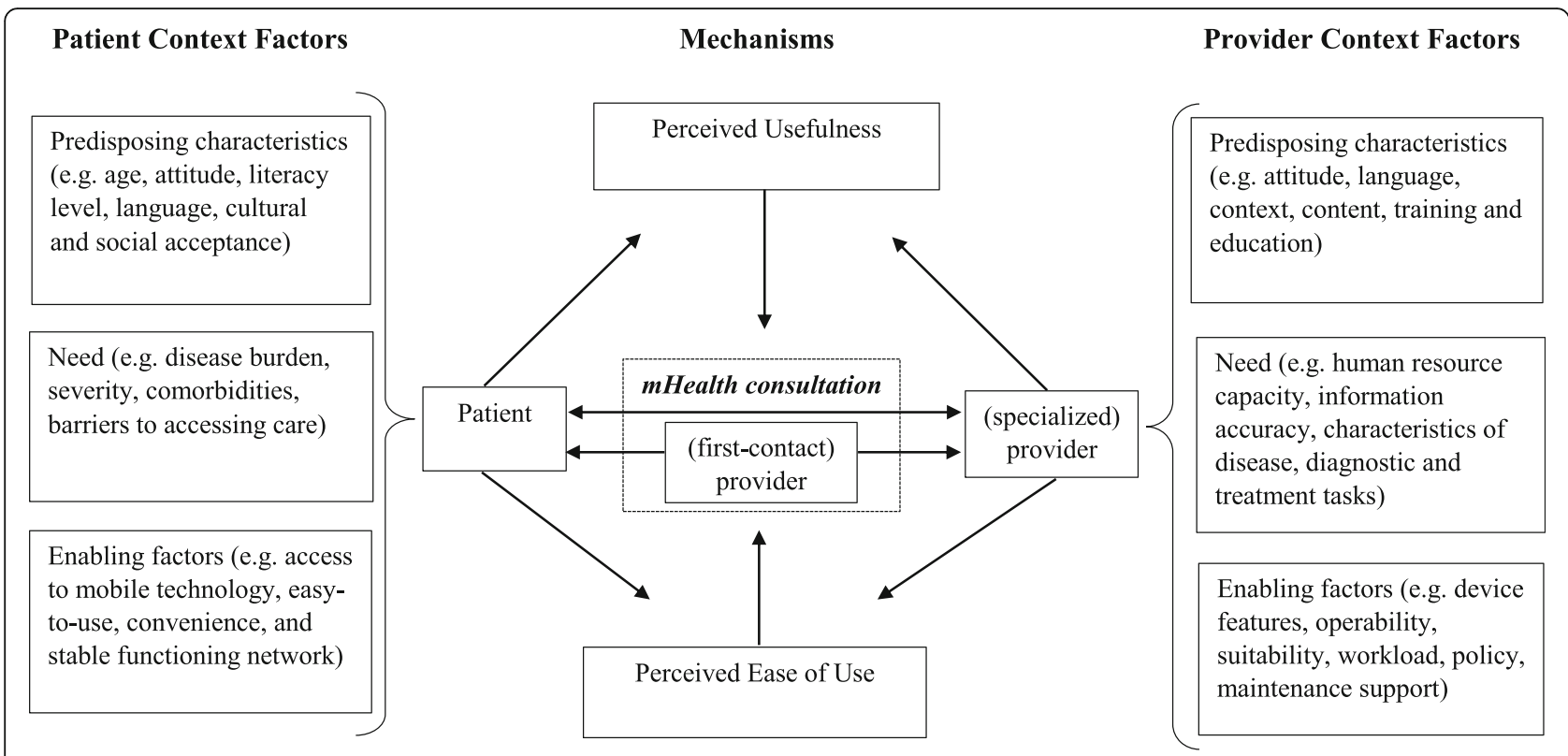

Fig. 2 Framework for understanding the contribution of mHealth interventions to improved access to care for patients with NCDs in sub-Saharan Africa

they find the mHealth intervention in comparison with other forms of service delivery, such as traditional faceto-face contacts or alternative computer-based telemedicine. Similarly, these factors also influence the perceived ease of use of mHealth in comparison with other options for service delivery. If interventions are perceived to be useful and easy to use, this will lead to the sustained use of mHealth interventions and - in turn - to better patient access to care (see Table 1).

\section{Main findings from the literature}

Table 2 summarizes the main findings from the literature, using the framework described above. It specifies separately for patients, (first-contact) providers, and (specialized) providers, what predisposing characteristics, enabling resources, and needs influence the perceived usefulness and the perceived ease of use.

\section{Predisposing characteristics}

For patients, the most important predisposing characteristic associated with the perception that a mHealth intervention was more useful than an alternative was the patients' cultural and social acceptance of the mobile technology, which involved familiarity with the technology in the community and absence of negative myths $[38,40,41,46$, 53, 55]. Other important predisposing characteristics of patients included positive attitudes toward the intervention and the ability to communicate in a comfortable language (see Table 2). Similar predisposing characteristics were also reported for providers, i.e., positive attitudes [38, 40,52], fluency in the language of the locality $[46,54]$, and sufficient training to use the technology $[47,52]$.
For both patients and (specialized) healthcare providers another important predisposing characteristic associated with the perception that mHealth was useful was source confidentiality [20, 39, 40, 49, 51]: Healthcare providers have to be confident that the information received via the mobile phone is accurate, and patients have to trust the (specialized) provider on the line in order to perceive the intervention as useful.

The perceived ease of use of an mHealth intervention depended most importantly on the predisposing characteristic that patients and providers were able to understand the language (see Table 2). In addition, studies reported that mHealth interventions have to be specifically designed to be easy to use for particular groups of patients, such as older age groups [55], or people with low educational levels $[50,55]$ or poor socio-economic backgrounds [50]. Firstcontact providers found mobile phone technologies easy to use if they were simple, relevant, and essentially combined local content and language [42]. Specialized providers' perception of ease of use was influenced by the accessibility of technical support, especially when there was the need to identify and solve technical problems such as software bugs [52].

\section{Need}

Patient needs were found to be particularly important factors influencing the perceived usefulness of mHealth interventions. If patients faced access barriers such as long travel times, waiting times, and high travel costs, mHealth interventions were perceived to be useful [20, 42, 45, 46, 48-50, 52, 53, 55]. Furthermore, three studies found that sicker patients were more likely to 


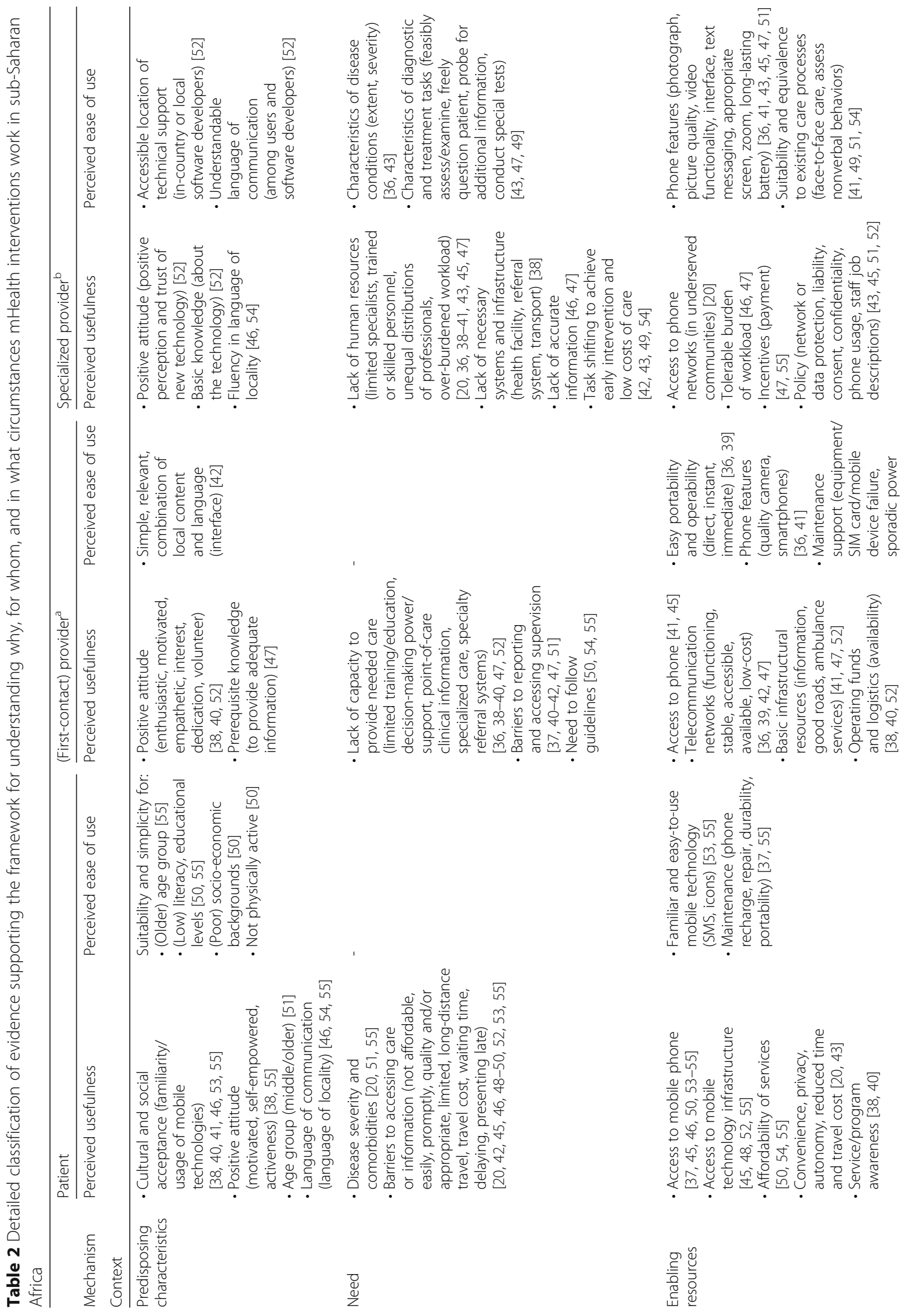


Opoku et al. BMC Medicine (2017) 15:24

Page 7 of 12

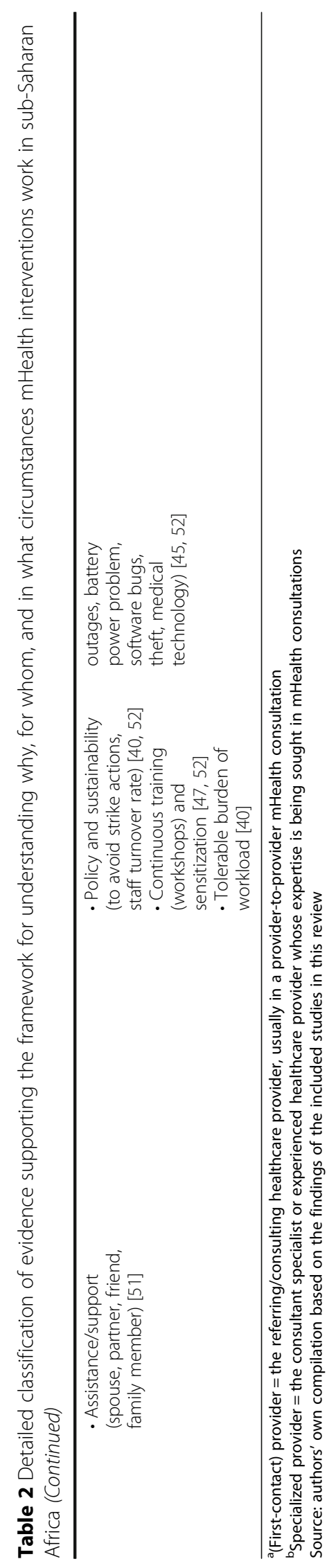


use the interventions, possibly because they found it easier to use the mHealth interventions rather than, for instance, walk to a provider $[20,51,55]$.

The most important need contributing to (first-contact) providers perceiving mHealth to be useful was their self-reported lack of capacity to provide adequate care [36, 38-40, 47, 52]. Furthermore, (firstcontact) providers reported that they needed support in order to follow guidelines [50, 54, 55] and that mHealth could contribute to overcoming barriers to accessing supervision [37, 40-42, 47, 51]. Also for (specialized) providers, several need factors contributed to the perceived usefulness of the intervention, including, for example, an over-burdening workload [20, 36, 38-41, 43, $45,47,50]$ and a lack of adequate referral and transport systems [38, 46, 47].

Studies did not report that the needs of patients and (first-contact) providers influenced their perceived ease of use. However, specialized providers found mHealth easier to use in the context of certain disease conditions, such as acne, herpes simplex, Kaposi's sarcoma, and flame burns in dermatology, than in others (scald burns, thickness wounds, and atopic dermatitis) [36, 43] and easier for certain diagnostic and treatment tasks (sharing feedback with patients, continuous clinical follow-ups) than for others (conducting physical examinations, special tests, and probing for additional information) $[43,47,49,51]$.

\section{Enabling resources}

For patients, unsurprisingly, the two most important enabling resources necessary for a mHealth intervention to be (perceived to be) useful were access to mobile phones [37, 45, 46, 50, 53-55] (also possible through borrowing $[46,50])$ and the availability of a functioning stable telecommunications network [20,36, 39, 42, 45, 47, 48, 52, 55]. Other enabling resources were assurance of privacy [43, 48], support from partners/relatives [51, 55], reduced costs of travel, and reduced time away from home or work [24, 50-53].

Enabling resources for first-contact providers were access to basic infrastructure, such as electric power and functioning medical technologies [49, 52], ambulance services and good roads [46, 47], as well as the affordability of telecommunication services and other operating costs $[50,54,55]$ (see Table 2). For (specialized) healthcare providers, the most important enabling resources were a tolerable additional workload [40,46, 47], the use of financial incentives $[47,55]$, and the availability of policy guidelines regarding data protection, phone usage, etc. [40, 43, 45, 52].

Enabling resources influencing patients' perceived ease of use of mHealth interventions included the durability and portability of mobile phones [37, 55] and the low complexity of the technology, for example, short message service (SMS) and icons [53, 55]. The same enabling resources - easy portability and operability [36, 39], using technologies from basic SMS to smartphones, built-in camera, and battery-saving apps $[41,43,45,52]$ - were also found to be associated with the perception among healthcare providers that mHealth was easy to use.

\section{Discussion}

\section{Summary of main findings}

This is the first realist review of mHealth interventions for patients with NCDs in SSA countries. It shows on the basis of a wide range of included studies how, for whom, and in what circumstances mHealth interventions contribute to improved access to (specialized) care for patients with NCDs in SSA. The review did not focus on specific interventions, specific diseases, or specific providers. Instead, it adopted a middle-range perspective to identify how contextual factors influence the outcome of mHealth interventions in terms of improved access to care; in other words, how to identify $\mathrm{C}-\mathrm{M}-\mathrm{O}$ relationships.

Our framework for understanding mHealth interventions illustrates the causal mechanisms that explain how, for whom, and in what circumstances mHealth interventions facilitate access to care (see Fig. 2). As to how mHealth interventions facilitate access to care, a mHealth intervention will ultimately contribute to improved access to care only if it is perceived to be useful and easy to use. The framework therefore shows that predisposing characteristics and needs of patients and healthcare providers as well as enabling resources influence the perceptions of patients and providers that mHealth interventions are useful and easy to use.

Considering for whom or how patients and provider characteristics influence mHealth interventions, the reviewed studies revealed that a positive attitude toward the mobile technology and the ability to communicate in a common language were the most important predisposing characteristics of patients and providers contributing to the perception that mHealth was useful and easy to use. In addition, needs of patients and providers, such as a high perceived burden of disease (e.g., in cases of reduced mobility) and the perceived lack of capacity of first-contact providers to provide adequate care, influenced the perceived usefulness and ease of use.

Furthermore, studies reported that certain circumstances of enabling resources, such as the availability of a stable communications network, accessible maintenance services, and regulatory policies (e.g., on data protection), contribute to the perception of patients and providers that mHealth interventions are useful and easy to use. 


\section{Strengths and implications for policy makers and program managers}

This review has several strengths. Following a realist methodology, it has included a wider scope of evidence than previous reviews $[6,10]$, and it has focused on the policy-relevant questions of how, for whom, and in what circumstances mHealth interventions facilitate access to care. The framework presented in Fig. 2 and the more specific context factors summarized in Table 2 have major implications for policy makers and program managers.

Firstly, given that predisposing characteristics of patients and providers influence the success of mHealth interventions, it is important that these factors are taken into account during the planning stages prior to the introduction of a new mHealth intervention. For example, program managers should consider evaluating the cultural and social acceptance among patients and providers to use the mobile technology when selecting a particular setting for the intervention. In particular, healthcare providers should be recruited who are enthusiastic and motivated to use mHealth as part of their job. Furthermore, interventions should be designed in such a way that patients, providers, and technical support will be able to communicate in a common language; otherwise, interventions are unlikely to be perceived to be useful and easy to use.

Secondly, and similar to the first point, it is important for policy makers and program managers to consider the specific needs of patients and (first-contact) providers to access (specialized) healthcare providers when preparing for the introduction of an mHealth intervention. For example, mHeath interventions will be particularly useful for severely ill patients or patients who face barriers to access (specialized) care, e.g., because they have difficulties in walking. Similarly, those (first-contact) providers who have a particular need for advice and supervision for treating certain groups of patients will perceive mHealth to be particularly useful. In addition, the influence of need factors on the perceived ease of use of (specialized) providers should be considered when preparing the introduction of an mHealth intervention, e.g., that mHealth is better for sharing feedback and continuous follow-up than for special tests and for probing for additional information [43, 47, 49].

Thirdly, policy makers and program managers have to be aware that the availability of enabling resources is essential for the successful implementation of an mHealth intervention. Enabling resources include, for example, easy access to mobile phones/devices, a stable and accessible communications network, and access to basic infrastructural resources, such as roads and ambulance services, which are necessary for mHealth supported referral systems [20, 36, 37, 39, 41, 42, 45-48, 50, 52-55]. Furthermore, policies on data protection and policies limiting the extra workload of mHealth interventions for professionals, possibly providing additional financial incentives, can support the sustained use of mHealth. See the checklist for policy guidance in Table 3.

\section{Limitations}

This review has a number of limitations. First, it does not answer the question of whether mHealth interventions facilitate improved access to care for patients with NCDs. It therefore does not contribute to the debate of whether mHealth interventions should be scaled up. Second, given that this review included a broad range of studies with various study designs, the inclusion of a specific study's finding into the review depended on rather subjective judgments. Following guidelines for realist reviews $[11,12,26,27]$, it was necessary to make decisions about whether a study's findings were relevant for the development of the framework and whether inferences drawn by an original study were sufficiently supported by evidence. Third, despite an extensive literature search and the inclusion of a wide range of studies, the available evidence on mHealth interventions in SSA remains rather limited. Therefore, the contextual factors summarized in Table 2 are rather indicative. It is very likely that there are further predisposing characteristics, enabling resources, and needs that are relevant for explaining how, for whom, and in what circumstances mHealth interventions work beyond those identified in our review. Future research is needed to confirm the theoretical framework developed in this paper and to operationalize some of its categories. For example, concerning the interplay of predisposing characteristics and perceived usefulness (see Table 2), research is needed to confirm that cultural and social acceptance is a predictor of perceived usefulness. This requires an operationalization for measuring cultural and social acceptance and for quantifying its impact on the sustained use of mHealth. Similarly, more research is necessary to better understand the interplay between need and specialized providers' ease of use. For example, researchers should explore the suitability of mHealth applications for different diseases and concerning different diagnostic and treatment tasks. This could include an assessment of the ease of use of mHealth for sharing feedback with patients with different diseases or different levels of severity, e.g., diabetes versus hypertension or diabetes with and without complications, and the differential effects on health outcomes.

\section{Conclusions}

The implementation of mHealth interventions in SSA has great potential to improve treatment and care for 
Table 3 A checklist for guiding the selection, development, implementation, evaluation, and policies regarding mHealth for treatment and care of non-communicable diseases in sub-Saharan Africa

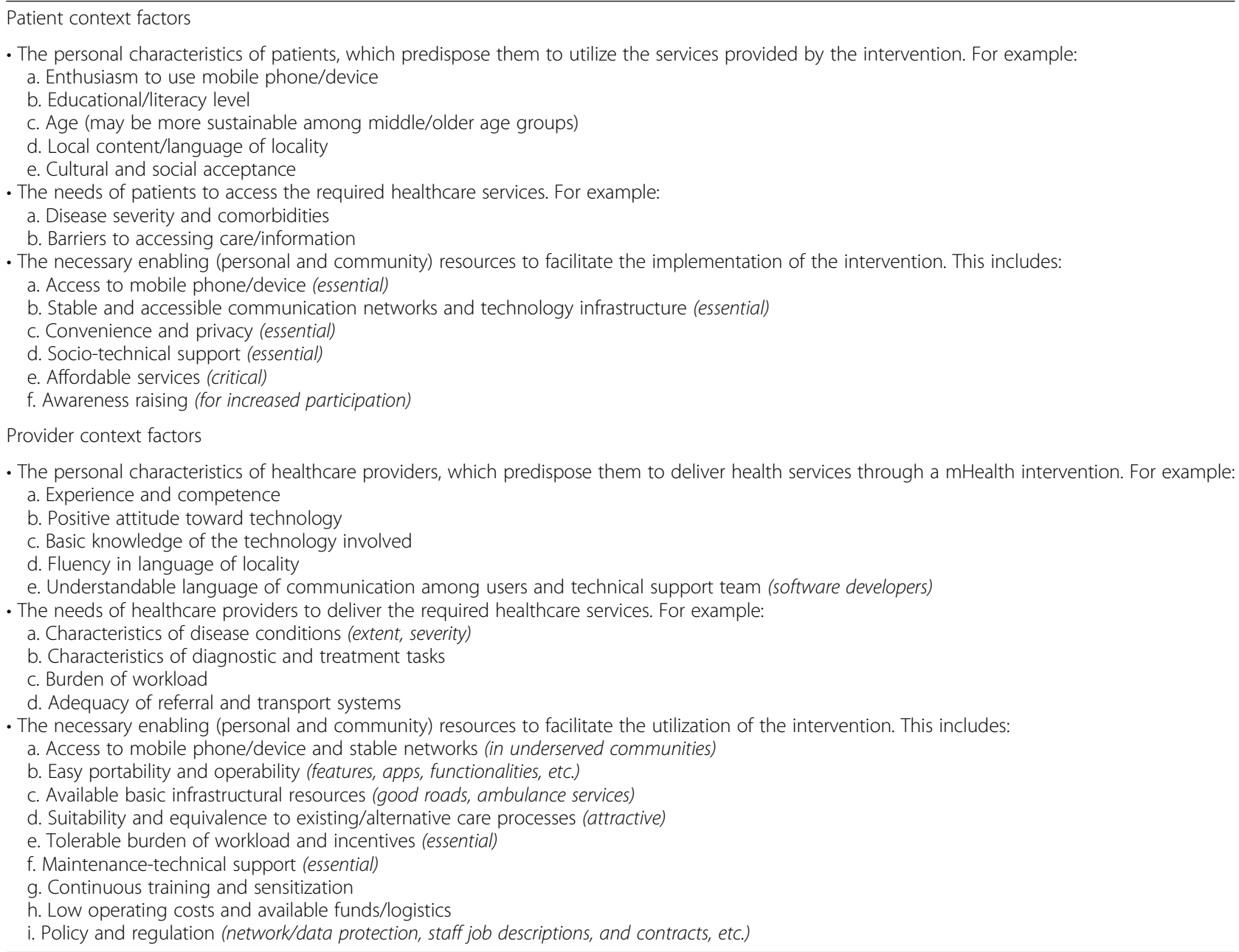

patients with NCDs, but the causal mechanisms explaining why, how, for whom, and in what circumstances these interventions work used to be unexplored. Our realist review shows that the contribution of mHealth interventions to improved treatment and care for patients with NCDs is that they facilitate (remote) access to previously unavailable and often specialized - services. In addition, our framework for understanding mHealth interventions illustrates that predisposing characteristics and needs of patients and healthcare providers as well as the availability of enabling resources in the community influence the perceptions of patients and providers that mHealth interventions are useful and easy to use - and these perceptions are essential for the successful implementation of an mHealth intervention.

The implication of these findings for policy makers and program managers is that predisposing characteristics and needs of patients and providers as well as the necessary enabling resources should be considered during the planning stages prior to the introduction of an mHealth intervention. In addition, researchers would benefit from placing greater attention on the context in which mHealth interventions are being implemented - as the context largely determines the predisposing characteristics and needs of patients and providers as well as the enabling resources - instead of focusing (too strongly) on the technical aspects of these interventions.

\section{Additional files}

Additional file 1: List of databases accessed and the search strategies used. (DOCX 20 kb)

Additional file 2: Theories included or excluded in review. (ZIP $28 \mathrm{~kb}$ )

Additional file 3: Description of the included articles providing the information for the synthesis and conclusions in this review. (DOCX $24 \mathrm{~kb}$ )

\section{Abbreviations}

C-M-O: Context-Mechanism-Outcome; mHealth: mobile phone-based health; NCD: non-communicable disease; SSA: sub-Saharan Africa; WHO: World Health Organization 


\section{Acknowledgements}

This study is part of a doctoral research project, and we thank Paul and Maria Kremer-Stiftung for awarding DO a scholarship. We thank Prof. Reinhard Busse for providing advice/guidance to the research, Ruth Waldherr for providing some papers used in the methods section, Greici Weinzierl for assisting with the translation of some papers, and Dr. Peter Agyei-Baffour and Dr. Anthony K. Edusei for their helpful comments on a draft of this review.

\section{Funding}

No funding was provided.

\section{Authors' contributions}

DO and WQ conceived and designed the study. DO and VS collected data. DO prepared an initial draft of the manuscript, which was subsequently revised by VS and WQ. All authors participated in data synthesis and analysis, and all read and approved the final manuscript.

\section{Competing interests}

The authors declare that they have no competing interests

\section{Consent for publication}

Not applicable.

\section{Ethics approval and consent to participate}

Not applicable.

Received: 11 August 2016 Accepted: 9 January 2017

Published online: 06 February 2017

\section{References}

1. Institute for Health Metrics and Evaluation HDN, The World Bank. The global burden of disease: generating evidence, guiding policy — sub-Saharan Africa regional edition. Seattle, WA: IHME; 2013.

2. Sambo LG. Health systems and primary health care in the African region. Afr Health Monit. 2011;(Special Issue 14):2-3.

3. Patrick Gold O, Ufuoma JE. Accelerating empowerment for sustainable development: the need for health systems strengthening in sub-Saharan Africa. Am J Public Health Res. 2013;1(7):152-8

4. Aranda-Jan CB, Mohutsiwa-Dibe N, Loukanova S. Systematic review on what works, what does not work and why of implementation of mobile health (mHealth) projects in Africa. Public Health. 2014;14:188.

5. Betjeman TJ, Soghoian SE, Foran MP. mHealth in sub-Saharan Africa. Int J Telemed Appl. 2013:2013:482324.

6. Bloomfield GS, Vedanthan R, Vasudevan L, Kithei A, Were M, Velazquez EJ. Mobile health for non-communicable diseases in sub-Saharan Africa: a systematic review of the literature and strategic framework for research. Global Health. 2014:10:49.

7. WHO. Global Action Plan for the prevention and control of noncommunicable diseases 2013-2020. Geneva, Switzerland: WHO Press, World Health Organization; 2013.

8. WHO. Atlas of eHealth country profiles: the use of eHealth in support of universal health coverage: based on the findings of the third global survey on eHealth 2015. Global Observatory for eHealth. Geneva, Switzerland: WHO Press, World Health Organization; 2016.

9. Stephani V, Opoku D, Quentin W. A systematic review of randomized controlled trials of mHealth interventions against non-communicable diseases in developing countries. BMC Public Health. 2016:16:572

10. Beratarrechea A, Lee AG, Willner JM, Jahangir E, Ciapponi A, Rubinstein A. The impact of mobile health interventions on chronic disease outcomes in developing countries: a systematic review. Telemed J E Health. 2014;20(1):75-82

11. Pawson R. Evidence-based policy: a realist perspective. London: Ashford Colour Press Ltd. SAGE Publications; 2006.

12. Wong G, Greenhalgh T, Westhorp G, Buckingham J, Pawson R. RAMESES publication standards: realist syntheses. BMC Med. 2013;11(21):14.

13. Kamanga A, Moono P, Stresman G, Mharakurwa S, Shiff C. Rural health centres, communities and malaria case detection in Zambia using mobile telephones: a means to detect potential reservoirs of infection in unstable transmission conditions. Malar J. 2010;9:96.
14. Wakadha H, Chandir S, Were EV, Rubin A, Obor D, Levine OS, et al. The feasibility of using mobile-phone based SMS reminders and conditional cash transfers to improve timely immunization in rural Kenya. Vaccine. 2013; 31(6):987-93.

15. Mbuagbaw L, Thabane L, Ongolo-Zogo P, Lester RT, Mills E, Volmink J, et al. The Cameroon mobile phone SMS (CAMPS) trial: a protocol for a randomized controlled trial of mobile phone text messaging versus usual care for improving adherence to highly active anti-retroviral therapy. Trials. 2011:12:5-5.

16. Leong KC, Chen WS, Leong KW, Mastura I, Mimi O, Sheikh MA, et al. The use of text messaging to improve attendance in primary care: a randomized controlled trial. Fam Pract. 2006;23(6):699-705.

17. Ostojic V, Cvoriscec B, Ostojic SB, Reznikoff D, Stipic-Markovic A, Tudjman Z. Improving asthma control through telemedicine: a study of short-message service. Telemed J E Health. 2005:11(1):28-35.

18. Shetty AS, Chamukuttan S, Nanditha A, Raj RK, Ramachandran A. Reinforcement of adherence to prescription recommendations in Asian Indian diabetes patients using short message service (SMS) — a pilot study. J Assoc Physicians India. 2011:59:711-4.

19. Wallis LA, Fleming J, Hasselberg M, Laflamme L, Lundin J. A smartphone app and cloud-based consultation system for burn injury emergency care. PLOS ONE. 2016;11(2):e0147253.

20. Azfar RS, Weinberg JL, Cavric G, Lee-Keltner IA, Bilker WB, Gelfand JM, et al. HIV-positive patients in Botswana state that mobile teledermatology is an acceptable method for receiving dermatology care. J Telemed Telecare. 2011;17:338-40

21. Ngabo F, Nguimfack J, Nwaigwe F, Mugeni C, Muhoza D, Wilson DR, et al. Designing and implementing an innovative SMS-based alert system (RapidSMS-MCH) to monitor pregnancy and reduce maternal and child deaths in Rwanda. Pan Afr Med J. 2012;13:31.

22. Andreatta P, Debpuur D, Danquah A, Perosky J. Using cell phones to collect postpartum hemorrhage outcome data in rural Ghana. Int J Gynaecol Obstet. 2011;113(2):148-51.

23. Greenhalgh TK, Kristjansson E, Robinson V. Realist review to understand the efficacy of school feeding programmes. BMJ Case Rep. 2007;335. doi: http://dx.doi.org/10.1136/bmj.39359.525174.AD.

24. TPM Editors. A reality checkpoint for mobile health: three challenges to overcome. PLoS Med. 2013;10(2):e1001395.

25. Craig P, Dieppe P, Macintyre S, Michie S, Nazareth I, Petticrew M. Developing and evaluating complex interventions: the new Medical Research Council guidance. BMJ. 2008, 337(a1655). doi:http://dx.doi.org/10.1136/bmj.a1655.

26. Pawson RG, Greenhalgh T, Harvey G, Walshe K. Realist review - a new method of systematic review designed for complex policy interventions. J Health Serv Res Policy. 2005;10 Suppl 1:21-34.

27. Wong G, Westhrop G, Pawson R, Greenhalgh T. Realist synthesis: RAMESES training materials. University of Oxford: RAMESES Project; 2013.

28. Riegel B, Jaarsma T, Stromberg A. A middle-range theory of self-care of chronic illness. ANS Adv Nurs Sci. 2012;35(3):194-204.

29. Fishbein M. A reasoned action approach to health promotion. Med Decis Making. 2008;28(6):834-44.

30. Rosenstock IM, Strecher VJ, Becker MH. Social learning theory and the Health Belief Model. Health Educ Q. 1988;15(2):175-83.

31. Andersen R. A behavioral model of families's use of health services. Research series: Center for Health Administration Studies. Chicago: University of Chicago; 1968. p. 25.

32. Andersen RM. Revisiting the behavioral model of and access to medical care: does it matter? J Health Soc Behav. 1995;36(March):1-10.

33. Rebhan PD. Health Care Utilization: Understanding and applying theories and models of health care seeking behavior. Case Western Reserve University, Ohio.

34. Chib A, van Velthoven MH, Car J. mHealth adoption in low-resource environments: a review of the use of mobile healthcare in developing countries. J Health Commun. 2015;20(1):4-34.

35. Davis FD. Perceived usefulness, perceived ease of use, and user acceptance of information technology. MIS Q. 1989;13(3):319-240.

36. Azfar RS, Lee RA, Castelo-Soccio L, Greenberg MS, Bilker WB, Gelfand JM, et al. Reliability and validity of mobile teledermatology in human immunodeficiency virus-positive patients in Botswana: a pilot study. JAMA Dermatol. 2014;150(6):601-7.

37. Chindo L. Mobile phone use in Cameroon: an increasingly useful tool for the follow-up of children with Burkitt lymphoma. Pediatr Blood Cancer. 2013;60(3):524 
38. Fiander A, Ndahani C, Mmuya K, Vanneste T. Results from 2011 for the transportMYpatient program for overcoming transport costs among women seeking treatment for obstetric fistula in Tanzania. Int J Gynaecol Obstet. 2013;120(3):292-5.

39. Fruhauf J, Hofman-Wellenhof R, Kovarik C, Mulyowa G, Alitwala C, Soyer HP, et al. Mobile teledermatology in sub-Saharan Africa: a useful tool in supporting health workers in low-resource centres. Acta Derm Venereol. 2013;93(1):122-3.

40. Greisman L, Nguyen TM, Mann RE, Baganizi M, Jacobson M, Paccione GA, et al. Feasibility and cost of a medical student proxy-based mobile teledermatology consult service with Kisoro, Uganda, and Lake Atitlan, Guatemala. Int J Dermatol. 2014. doi:10.1111/ijd.12708.

41. Holeman I, Evans J, Kane D, Grant L, Pagliari C, Weller D. Mobile health for cancer in low to middle income countries: priorities for research and development. Eur J Cancer Care (Engl). 2014;23(6):750-6.

42. Kingue S, Angandji P, Menanga AP, Ashuntantang G, Sobngwi E, Dossou-Yovo RA, et al. Efficiency of an intervention package for arterial hypertension comprising telemanagement in a Cameroonian rural setting: the TELEMED-CAM study. Pan Afr Med J. 2013;15:153.

43. Kiser M, Beijer G, Mjuweni S, Muyco A, Cairns B, Charles A. Photographic assessment of burn wounds: a simple strategy in a resource-poor setting. Burns. 2013;39(1):155-61.

44. Goldbach H, Chang AY, Kyer A, Ketshogileng D, Taylor L, Chandra A, et al. Evaluation of generic medical information accessed via mobile phones at the point of care in resource-limited settings. J Am Med Inform Assoc. 2014;21:37-42.

45. Ndlovu K, Littman-Quinn R, Park E, Dikai Z, Kovarik CL. Scaling up a mobile telemedicine solution in Botswana: keys to sustainability. Front Public Health. 2014;2:275.

46. Odigie VI, Yusufu LM, Dawotola DA, Ejagwulu F, Abur P, Mai A, et al. The mobile phone as a tool in improving cancer care in Nigeria. Psychooncology. 2012;21(3):332-5.

47. Opoku D, Scott P, Quentin W. Healthcare professionals' perceptions of the benefits and challenges of a teleconsultation service in the Amansie-West District of Ghana. Telemed J E Health. 2015. doi:10.1089/tmj.2014.0210.

48. Osei-tutu A, Shih T, Rosen A, Amanquah N, Chowdhury M, Nijhawan RI, et al. Mobile teledermatology in Ghana: sending and answering consults via mobile platform. J Am Acad Dermatol. 2013;69(2):e90-91.

49. Qin R, Dzombak R, Amin R, Mehta K. Reliability of a telemedicine system designed for rural Kenya. J Prim Care Community Health. 2013:4:177-81.

50. Stewart A, Noakes T, Eales C, Shepard K, Becker P, Veriawa Y. Adherence to cardiovascular risk factor modification in patients with hypertension. Cardiovasc J S Afr. 2005;16(2):102-7.

51. Temmingh $\mathrm{H}$, Claassen A, van Zyl S, Carrara H, Dayakalashe NM, Myer L, et al. The evaluation of a telephonic wellness coaching intervention for weight reduction and wellness improvement in a community-based cohort of persons with serious mental illness. J Nerv Ment Dis. 2013;201:977-86.

52. Littman-Quinn R, Mibenge C, Antwi C, Chandra A, Kovarik CL. Implementation of $\mathrm{m}$-health applications in Botswana: telemedicine and education on mobile devices in a low resource setting. J Telemed Telecare. 2013:19:120-5.

53. Kivuti-Bitok LW, McDonnell G, Pokhariyal GP, Roudsari AV. Self-reported use of internet by cervical cancer clients in two National Referral Hospitals in Kenya. BMC Res Notes. 2012;5:559.

54. Pastakia SD, Karwa R, Kahn CB, Nyabundi JS. The evolution of diabetes care in the rural, resource-constrained setting of western Kenya. Ann Pharmacother. 2011;45:721-6.

55. Rotheram-Borus MJ, Tomlinson M, Gwegwe M, Comulada WS, Kaufman N, Keim M. Diabetes buddies: peer support through a mobile phone buddy system. Diabetes Educ. 2012;38:357-65.

\section{Submit your next manuscript to BioMed Central and we will help you at every step:}

- We accept pre-submission inquiries

- Our selector tool helps you to find the most relevant journal

- We provide round the clock customer support

- Convenient online submission

- Thorough peer review

- Inclusion in PubMed and all major indexing services

- Maximum visibility for your research

Submit your manuscript at www.biomedcentral.com/submit
Biomed Central 